\title{
Identification of BAF57 mutations in human breast cancer cell lines
}

Evangelos Kiskinis $^{1}$, Juana M. García-Pedrero ${ }^{1}$, M. Angeles Villaronga ${ }^{2}$, Malcolm G. Parker $^{1}$ and Borja Belandia ${ }^{2 *}$

${ }^{1}$ Institute of Reproductive and Developmental Biology, Imperial College London, Du Cane Road, London, W12 ONN, United Kingdom

${ }^{2}$ Instituto de Investigaciones Biomédicas, Consejo Superior de Investigaciones Científicas and Universidad Autónoma de Madrid, Arturo Duperier 4, 28029 Madrid, Spain

Running title: BAF57 mutations in breast cancer cells

\section{*Corresponding author:}

Borja Belandia: Tel: +34 91585 4453, Fax: +34 91585 4401, e-mail: bbelandia@,iib.uam.es

Key words: BAF57, breast cancer cells, chromatin remodeling, estrogen receptor, SWI/SNF. 


\section{Summary}

Accumulating genetic and biochemical evidences support a role for the SWI/SNF chromatin remodeling complex in cancer development and multiple core subunits of these complexes have been found to function as tumor suppressor genes. The core SWI/SNF subunit BAF57 mediates direct interactions with estrogen and androgen receptors (ER and AR) regulating their transcriptional activity. BAF57 gene maps to chromosome band 17q21 in close proximity to the $B R C A 1$ gene. This locus has been associated with frequent loss of heterozygosity $(\mathrm{LOH})$ and allelic imbalance in breast cancers; however $B R C A 1$ mutations are rare events in sporadic breast cancer with $\mathrm{LOH}$ in the region, suggesting that another tumor suppressor gene resides in this area. All these reasons prompted us to screen for mutations in the BAF57 gene using a panel of the most commonly used human breast cancer cell lines. All cell lines analyzed contain wild-type copies of BAF57 gene with the only exception of the breast ductal carcinoma cell line BT549. Sequencing of genomic DNA and cDNA generated from BT549 mRNA demonstrated the presence of a CA dinucleotide insertion in exon 5 of BAF57. The absence of wild-type BAF57 alleles indicates that this is a biallelic inactivating mutation that causes a frameshift and as a consequence a premature stop codon leading to a truncated BAF57 protein. A functional characterization of the truncated BAF57 showed that it has lost the ability to bind to ER but still binds to the nuclear receptor coactivator SRC1e. Furthermore, we observed that the expression of the truncated BAF57 increased the ability of SRC1e to potentiate transcriptional activation by ER $\alpha$, suggesting that mutations in BAF57 could contribute to the oncogenic transformation in breast cancer cells. 


\section{Introduction}

Estrogens play a central role in mammary gland development and morphogenesis but they also are important in the proliferation and progression of breast cancer (reviewed in [1]). Although there is growing evidence that estrogens can act through nongenomic pathways most described estrogen actions are mediated by the estrogen receptor alpha $(E R \alpha)$ and beta $(E R \beta)$ acting as ligand-dependent transcription factors. Estrogen receptors belong to the nuclear receptor superfamily [2] and they regulate many physiological processes in response to its natural ligand, 17ß-estradiol (E2). Endocrine therapy with ER antagonists, and specially tamoxifen, is the most effective systemic treatment for all stages of ER-positive breast cancer. However, many patients present primary resistance to endocrine therapy despite expressing high levels of ER and all patients with advance breast cancer eventually develop resistance to therapy ([3] and references therein). To understand the basis of the appearance of this resistance we need to elucidate the detailed molecular mechanisms that regulate ER activity.

Upon ligand binding, ERs bind to specific DNA sequences (EREs) present in the promoters of estrogen-dependent genes, triggering the recruitment of the enzymatic activities responsible for the regulation of gene expression. To overcome the repressive environment that represents the tightly packed chromatin fiber, ERs recruit many chromatin

remodeling enzymes that can be divided into two distinct classes; first, ATP-dependent chromatin remodeling complexes that use the energy of ATP hydrolysis to modify the location and association of nucleosomes with DNA, and second, enzymes that catalyze covalent posttranslational modifications in histones. These proteins are named collectively 
nuclear receptor coregulators and they act in a concerted manner contributing to the reorganization of the chromatin templates and the recruitment of basal transcription factors and RNA polymerase II [4].

Several studies have demonstrated that mammalian SWI/SNF, an ATP-dependent chromatin remodeling complex, can enhance the transcriptional activity of the ER and several other nuclear receptors [5-10]. The SWI/SNF family comprises a number of large multiprotein complexes containing between eight to ten subunits. These complexes always contain a central catalytic subunit, BRM/SNF $\alpha$ or BRG1/SNF $\beta$, and several other variable BRG1-associated factors (BAFs) that contribute to the enzymatic activity of the complex and facilitate the recruitment to specific transcription factors although the detailed functions of each individual subunit remain to be determined. Growing evidence indicates that SWI/SNF has a role in cancer development and several subunits either act as tumor suppressors or are required for the regulation of other tumor suppressor genes (Reviewed in $[11,12])$. For instance, the SWI/SNF complex has been shown to associate with BRCA1 through a direct interaction with BRG1/SNF $\beta$ subunit and mutations in BRG1/SNF $\beta$ abrogated stimulation of transcription by BRCA1 [13]. Mutations in the BRCA1 gene confer an increased risk for estrogen- and androgen-responsive cancers [14]. BRCA1 maps to chromosome band 17q21, a locus associated with frequent loss of heterozygosity (LOH) and allelic imbalance in breast cancers [15]. In sporadic breast cancer the $B R C A 1$ gene is rarely mutated in samples with $\mathrm{LOH}$ in the locus [16], suggesting that other genes in this region might act as tumor suppressors. Interestingly, the gene that encodes BAF57, another SWI/SNF subunit, also maps to chromosome band 17q21. We have previously 
demonstrated that BAF57 interacts directly with ER and also with all members of the p160 family of nuclear receptor coactivators [10]. The interaction between BAF57 and ER is regulated by estrogen and BAF57 was shown to be essential for the coactivation of ER carried out by the p160 coactivators using a human breast ductal carcinoma cell line (BT549) reported to be negative for the BAF57 protein [17]. Moreover, BAF57 was recruited to estrogen-responsive promoters in a ligand-dependent manner, suggesting that the in vivo functions of ER as transcription factor might depend on the recruitment of SWI/SNF complexes by means of an interaction with BAF57. A previous report analyzed the BAF57 protein status in a number of human cell lines commonly used as in vitro models in breast cancer research [17]. In light of the new data reinforcing a role for BAF57 in the control of ER activity, a key regulator of cell proliferation in estrogen-responsive tumors, we have extended those studies by sequencing the BAF57 gene in a panel of human breast cancer cell lines, looking for possible mutations that could have occurred during tumor progression and/or the establishment of the cell line that might affect normal ER-dependent signaling. Here we report our findings describing the first mutation characterized in the human $B A F 57$ gene and discuss its possible implications in the development of breast cancer. 


\section{Materials and methods}

\section{Cell culture}

Carcinoma-derived cells were grown as a monolayer on $75 \mathrm{~cm}^{2}$ tissue culture flasks at $37^{\circ} \mathrm{C}$ in a humid atmosphere maintained at $10 \%(\mathrm{v} / \mathrm{v}) \mathrm{CO}_{2}$. Cells were maintained in either DMEM (ZR-75-1, MCF-7, T47D, MDA-MB-361, MDA-MB-231, PC-3) or RPMI 1640 (BT549 and LNCaP) supplemented with 10\% (v/v) fetal bovine serum.

\section{Plasmids}

The mutated BAF57 open reading frame found in BT549 cells was amplified by PCR and subcloned into pSG5 (pSG5-BT-BAF57) or pGEX-6P-1 (Amersham Pharmacia Biotech, GST-BT-BAF57).

The following plasmids have been described previously: pMT2-MOR, pSG5-SRC1e, pGL3-2ERE-PS2-LUC, pSG5- $\Delta$ N-BAF57, GST-BAF57 [10].

\section{Genomic Extraction}

The DNeasy tissue kit (Qiagen) was used for rapid isolation of total genomic DNA from cultured cells. Cells were grown to $50 \%$ confluency in a $9 \mathrm{~cm}$ petri-dish (maximum number of cells $5 \times 10^{6}$ ), scraped off from the bottom of the petri dish, centrifuged at 1000 rpm for 5 min and resuspended in $200 \mu 1$ PBS. Total genomic DNA was isolated according to the manufacturer's protocol using two elution steps to yield DNA concentrations between $60-180 \mathrm{ng} / \mu \mathrm{l}$.

DNA sequencing 
DNA was sequenced by a linear PCR method using dye-labelled terminators from Applied Biosystems. Typically, sequencing reactions were set-up consisting of $1 \mu 1$ DNA, $8 \mu l$ of Big Dye Terminator Ready Mix 3 (containing Taq polymerase and dye-labelled terminators) and $3 \mu l(10 \mathrm{ng} / \mu \mathrm{l})$ of primer in a $20 \mu \mathrm{l}$ reaction. PCR reactions typically consisted of 25 thermo-cycles of: $94^{\circ} \mathrm{C}$ for 30 seconds, $50^{\circ} \mathrm{C}$ for 15 seconds and $60^{\circ} \mathrm{C}$ for 4 minutes. Following the PCR, the DNA was precipitated, washed and then processed using the ABI 3100 capillary sequencer and the collection data using the ABI 3100 data collection software.

\section{Real-Time RT-PCR}

The Invitrogen ready-to-use TRIZOL reagent was used to isolate total RNA from cultured cells $\left(>10^{7}\right)$. Precipitation and concentration of total RNA was achieved by the addition of isopropyl alcohol, centrifugation, and a subsequent $75 \%$ ethanol wash. Purified RNA was briefly air dried and re-dissolved in DEPC-treated water (Ambion). The RNA concentration and purity were determined by measurement of the $\mathrm{OD}_{260}$ and $\mathrm{OD}_{280}$ on a spectrophotometer. To obtain first strand cDNA, $2 \mu \mathrm{g}$ total RNA were treated with DNase I, and cDNA was prepared using the Superscript First-Strand synthesis System for RT-PCR according to the manufacturer's instructions (Life Technologies, Inc.).

Analysis of gene expression was performed by Real-time PCR using the ABI PRISM 7700 Sequence detector following PE Applied Biosystems' Taqman SYBR Green Master Mix protocol (Applied Biosystems, Foster City, CA). Reactions were carried out using specific primers for hBAF57 and the constitutively expressed L19 ribosomal coding gene 
used as an internal control. BAF57 expression levels were normalized to L19 levels. Primer sequences may be obtained on request.

\section{GST pull-down assays}

Expression vectors for $\mathrm{ER} \alpha$ or SRC1e-PAS were transcribed and translated in vitro

with $\left[{ }^{35} \mathrm{~S}\right]$-methionine in reticulocyte lysate (Promega) according to the manufacturer's protocol. GST fusion proteins of full-length BAF57 and truncated BT-BAF57 were induced, purified, bound to Sepharose beads (Amersham), and incubated with in vitro translated proteins (ER $\alpha$ or SRC1e-PAS) as described previously [10].

\section{Transient transfections}

COS-1 cells were cultured in DMEM supplemented with 10\% fetal bovine serum. Twenty-four hours before transfection, cells were plated in 24-well plates in phenol redfree medium, supplemented with 5\% dextran charcoal-stripped serum. Cells were transfected using a modified calcium phosphate protocol [18]. The transfected DNA included a pRL-CMV (Promega) control plasmid (5ng/well), the luciferase reporter plasmid pGL3-2XERE-PS2 (100 ng/well), pMT2-MOR (25 ng/well), pSG5-SRC1e (100 ng/well), pSG5- $\Delta$ N-BAF57 (250 ng/well) or pSG5-BT-BAF57 (250 ng/well). Empty vectors were used to normalize the amounts of DNA. After incubation for 16 hours with the DNAs, the cells were washed and incubated in fresh medium in the presence of $10 \mathrm{nM} 17 \beta-$ estradiol for $24 \mathrm{~h}$. Subsequently, cells were harvested and extracts were assayed as described previously [18]. 


\section{RESULTS}

Screening cancer cell lines for mutations in the BAF57 gene

In order to investigate the possibility that mutations in $B A F 57$ occur in human breast cancer cell lines that might affect ER transcriptional activity, we have screened a panel of ER positive and ER negative breast cancer cell lines for mutations in the BAF57 gene that might affect estrogen signaling. In addition we also analyzed the sequence of BAF57 gene in two of the most commonly used human prostate cancer cell lines, LNCaP and PC-3 (Table I). The BAF57 gene was sequenced directly from cDNA generated using RNA extracted from the cell lines MDA-MB-231, PC-3 and LNCaP and no mutations were found affecting BAF57 sequence. Genomic DNA was extracted from the other five cell lines studied and eleven sets of primers (forward and reverse) were used to both PCRamplify and sequence all eleven exons of BAF57. From all the cell lines sequenced, we found a CA dinucleotide insertion located in exon 5 of BAF57 derived from the BT549 breast cancer cell line. The 5'-CA-3' insertion was detected in two sequencing reactions with both primers from two independent PCR-amplification reactions of exon 5 (Figure 1a). The presence of single peaks and no overlaps in the DNA sequencing suggests that the CA insertion is either a homozygous mutation or only one mutated allele is present.

A restriction enzyme map analysis of BAF57 exon 5 showed that the CA mutation disrupted a unique $R s a$ I restriction endonuclease site (GT/AC). Digestion of the wild-type sequence with this enzyme generates two fragments of approximately equal length (Figure 1b). In order to confirm the presence of the CA mutation, exon 5 amplicons were PCRamplified using genomic DNA from BT549 and from MCF-7 (reference control with two 
wild-type copies of BAF57 gene) and were digested with the $R s a$ I endonuclease. The sizes of the products obtained in the digestion reactions were analyzed by agarose gel electrophoresis (Figure 1c). Clearly, the presence of the CA mutation in the DNA derived from BT549 cells disrupted the $R s a$ I restriction site, resulting in the appearance of a single 512-bp band in the gel corresponding to the undigested exon 5 amplicon, while DNA from MCF-7 only containing wild-type BAF57 copies gave rise to two fragments of 265 and 247-bp that run as a single band in this $1.2 \%$ gel. The detection of only a single 512-bp band in the BT549-derived amplicon confirmed that BT549 do not have any wild-type BAF57 allele.

\section{Characterization of the novel BAF57 mutation found in BT549 cells}

We next investigated the consequences of the BT549 mutation at the protein level. Using the DNA Strider software we analyzed how the CA insertion at exon 5 affects the open reading frame (ORF) of BAF57 sequence and consequently the translation of the protein (Figure 2). The wild-type protein consists of 411 amino acids (Figure 2a), while as a result of the CA dinucleotide insertion a premature stop codon is introduced at nucleotide 250 giving rise to a truncated protein that contains the first 75 amino acids of BAF57, followed by 9 random amino acids (Figure 2b). Furthermore this provides with an explanation to why in a previous study DeCristofaro et al. failed to detect full-length BAF57 protein in BT549 cells by Western blot analysis [17], since the mutation that we have identified in this breast cancer cell line generates a truncated BAF57 protein.

In order to examine whether the breast ductal carcinoma cell line BT549 expressed the mutated BAF57 mRNA encoding the truncated protein, we isolated total RNA, generated 
cDNA and mRNA levels were monitored by Real-Time RT-PCR (Figure 3). The relative expression levels of BAF57 were analyzed in a number of breast and prostate carcinomaderived cell lines (BT549, ZR-75-1, MCF-7, T47D, LNCaP and PC-3) and we found detectable BAF57 mRNA expression in BT549 cells. However, it is noteworthy that BT549 cells express significantly lower BAF57 mRNA levels compared to the rest of cell lines examined. The presence of the CA mutation was also confirmed by sequencing the cDNA generated from BT549 cells (data not shown).

In an attempt to characterize the functional effects of the BT549-derived BAF57 (BTBAF57) truncated protein, we subcloned the mutated ORF into the bacterial expression vector pGEX-6P1 and the mammalian expression vector pSG5, to be used in GST pulldown assays and transient transfection experiments respectively.

We have previously demonstrated that BAF57 directly interacts with both ER $\alpha$ and p160 coactivators and this association is required for the ability of p160 coactivators to potentiate ER-mediated transcription in transient transfection systems [10]. The CA insertion leads to a truncated BAF57 protein what could have important consequences on the functionality of this protein. We carried out GST pull-down assays to investigate the ability of the truncated BT-BAF57 protein to interact in vitro with both ER $\alpha$ and the p160 coactivator SRC1e, as compared to wild-type full-length BAF57. In a set of GST pull-down experiments in vitro translated ${ }^{35} \mathrm{~S}$-labelled ER $\alpha$ was incubated with GST-BAF57 (wildtype full-length BAF57) or GST-BT-BAF57 (truncated BAF57) fusion proteins (Figure 4a). According to our previous data, we observed that ER $\alpha$ directly binds to GST-BAF57 in a ligand-dependent manner with a strong interaction detected in the presence of estrogen 
(E2) (Figure 4a). However, the in vitro binding of ER $\alpha$ to GST-BT-BAF57 was dramatically impaired (Figure 4a). No interaction was observed in the presence of GST alone, as a proof of the specificity of BAF57-ER $\alpha$ interaccion. In another set of experiments we analyzed the in vitro interaction between the bHLH-PAS domain (amino acids 1-361) of SRC1e (responsible for mediating the interaction with BAF57) and GSTBAF57 or GST-BT-BAF57 fusion proteins (Figure 4b). In vitro translated ${ }^{35}$ S-labelled PAS-SRC1e interacted in the same weak but specific manner with both full-length BAF57 and BT-BAF57. Collectively the data from the in vitro pull-down assays indicate that although the BT-BAF57 still retains its ability to interact with p160 coactivators similar to that of full-length BAF57, this truncated protein lacks capability of binding ER $\alpha$.

Next we investigated the possible functional consequences of the expression of the truncated BAF57 protein in ER $\alpha$ transcriptional activity. We and others have demonstrated that several truncated BAF57 proteins act as dominant-negative forms on steroid receptor dependent transcriptional activity when expressed in cells containing endogenous BAF57 $[10,20]$. To test whether the truncated BT-BAF57 was able to modulate the transcriptional activity of ER $\alpha$ we examined transcription from the 2ERE-PS2-LUC reporter in the presence of the $\mathrm{ER} \alpha, \mathrm{SRC} 1 \mathrm{e}$ and either the previously characterized dominant negative $\Delta$ N-BAF57 or the truncated BT-BAF57 cloned from BT549 cells. As previously reported $\triangle$ N-BAF57 dramatically inhibited the ability of SRC1e to potentiate transcriptional activity of ER $\alpha$ (Figure 4c). Surprisingly, the expression of BT-BAF57 did not reduce SRC1e coactivation potential; on the contrary, we observed an increase in the SRC1e coactivation of ER $\alpha$ in the presence of BT-BAF57 (Figure 4c). 


\section{Discussion}

The considerable genetic and biochemical evidence linking BAF57 to cancer prompted us to screen a number of breast and prostate cancer cell lines for mutations in the BAF57 gene. The human BAF57 gene maps to chromosome band $17 \mathrm{q} 21$, in close proximity to the $B R C A 1$ gene, a locus associated with frequent $\mathrm{LOH}$ and allelic imbalance in breast, ovarian and prostate cancer and it has previously been suggested that BAF57, based on its involvement in DNA transcription and repair events, could function as a tumor suppressor [17]. Moreover the involvement of BAF57 in the regulation of transcriptional activity by ER $\alpha$ [10], which is a key regulator of cell proliferation in normal as well as in cancerous breast and ovarian tissues, further reinforces this notion. In this study we address the characterization of BAF57 gene in the most widely used human cancer cell lines exploited as in vitro models in breast cancer research [21]. The experimental approach chosen was preferentially sequencing genomic DNA, because this allows us to detect mutations in $B A F 57$ even in cases where expression of the gene could be lost during cancer development or cell line establishment. Five out of six breast cancer cell lines studied do not contain mutations in BAF57 affecting the expressed protein or the DNA sequence. However, a CA dinucleotide insertion in BAF57 was found in the BT549 breast cancer cell line. The observation of single peaks and no overlaps in the electropherogram in the DNA sequence suggests that the wild-type BAF57 allele is not present in BT549 cells. A restriction digestion analysis of $B A F 57$ exon 5 further confirmed that these cells only contain mutated copies of BAF57. Therefore, either the CA insertion occurs in homozygosis or, more likely, according to the Knudson's two-hit model [22], the acquisition of this mutation in one copy 
of BAF57 was followed by the subsequent loss of the remaining wild-type copy of the gene. Although we do not have conclusive evidence several observations support this notion. During the PCR-amplification reactions we observed that for BT549 almost double the amount of genomic DNA was required, relative to that of other cell lines, in order to generate the same amount of amplified product. In addition, the results of the Real-Time RT-PCR, where it was shown that BT549 cells express significantly lower levels of BAF57 mRNA than the other cell lines further suggest the presence of a single BAF57 allele in this cell line, although we cannot rule out the possibility that other mechanisms, like promoter methylation, could also be responsible for this low expression. As mentioned above, BAF57 maps to a chromosome area that has been associated with $\mathrm{LOH}$ in breast cancer and our data suggest that this is the case in BT549 cells. The CA insertion located in BAF57 exon 5 causes a frameshift in the ORF and introduces a premature stop codon, leading to a truncated protein (BT-BAF57) consisting of eighty-five amino acids, of which only the first seventy-five correspond to wild-type BAF57 protein.

In view of the critical role of BAF57, acting through SWI/SNF complexes, in the regulation of gene transcription mediated by ER $\alpha$ and $\mathrm{AR}$, the confirmation that all other breast and prostate cancer cell lines analyzed express wild-type BAF57 might be relevant in future studies performed using those cellular models addressing the molecular mechanisms of action of SWI/SNF complexes, ER or AR.

BT549 is a breast-ductal carcinoma cell line that does not express ER and has a truncated retinoblastoma (pRB) transcript ([23] and references therein). A previous study had also shown that the BAF57 protein was undetectable by Western blotting in this cell 
line [17] and the mutation we identified in the $B A F 57$ gene that results in the generation of a truncated protein explains why. We have also shown that BT549 cells express BAF57 mRNA encoding the truncated protein. BT-BAF57 does not interact with ER but it is still able to bind in vitro to the SRC1e coactivator in the same manner as the full-length wildtype protein raising the possibility that the truncated protein may interfere not only with ER $\alpha$ regulation, but also with a number of other nuclear receptor-dependent gene regulation pathways. Alterations on AR transcriptional activity might also be relevant in the generation of the transformed phenotype in breast cancer cells because its activity requires both functional p160 coactivators and BAF57, and AR can mediate testoterone antiproliferative and proapoptotic effects in mouse breast cancer models (reviewed in [24])

In most cases cancer arises after an accumulation of mutations that may affect different cell proliferation-regulatory pathways. In the case of BT549, at least three mutations are present, each potentially linked to generation of breast cancer. The pRB protein is a major tumor suppressor and transfection studies have shown that the SWI/SNF complex associates with pRB to inhibit cell proliferation [25]. BT549 cells are also ER negative and estrogen is a major regulator of cell proliferation in normal as well as in breast cancer tissues. Finally, BAF57 is a core subunit of all human SWI/SNF complexes and a truncated BAF57 protein may potentially interfere with both pRB-mediated and ER $\alpha$-mediated pathways. We have observed that expression of BT-BAF57, in contrast to the reported dominant-negative action of other artificial BAF57 deletion mutants, is able to increase the ability of SRC1e to potentiate transcriptional activation by ER $\alpha$. Therefore, if this mutation occurred prior to the loss of ER expression, it could have contributed to the aberrant 
cellular proliferation through excessive estrogen-dependent signaling. It is also possible that the BAF57 mutation occurred first and pleiotropic effects mediated by deficient SWI/SNF complexes led to the generation of additional mutations. BAF57, acting through SWI/SNF complexes, participates in the regulation of gene expression carried out by many types of transcription factors other than ER and AR. A recent study has shown that stably expression of full-length wild-type BAF57 in BT549 cells reduced the transformed characteristics of this breast cancer cell line, inducing cell cycle arrest and apoptosis and restoring contact inhibition [26], strongly supporting a role for BAF57 as tumor suppressor. To address the possible contribution of this mutation in BAF57 to aberrant ER-dependent signaling it will be necessary to stably express BT-BAF57 mutant in cell lines expressing both wild-type BAF57 and ER in order to analyze how BT-BAF57 affects estrogen dependent gene expression and cell proliferation.

The finding of a mutation in BAF57 in one breast cancer cell line out of just six that were screened strengthens our view that BAF57 may be associated with tumor generation in the mammary gland and it suggests that it will be worthwhile to initiate studies to screen primary human breast cancer samples for potential mutations in the $B A F 57$ gene. 


\section{Acknowledgements}

We are grateful to Roman Mullenbach for his help in setting up the genomic sequencing assay. Our work was funded by the Wellcome Trust (grant 061930), the Association for International Cancer Research (03-098) and Ministerio de Educación y Ciencia (SAF2004-02549). 


\section{References}

1. Herynk MH, Fuqua SA: Estrogen receptor mutations in human disease. Endocr Rev 25: 869-898, 2004

2. Mangelsdorf DJ, Thummel C, Beato M, Herrlich P, Schutz G, Umesono K, Blumberg B, Kastner P, Mark M, Chambon P, Evans RM: The nuclear receptor superfamily: the second decade. Cell 83: 835-839, 1995

3. Jordan VC: Selective estrogen receptor modulation: concept and consequences in cancer. Cancer Cell 5: 207-213, 2004

4. McKenna NJ, O'Malley BW: Combinatorial control of gene expression by nuclear receptors and coregulators. Cell 108: 465-474., 2002

5. Yoshinaga SK, Peterson CL, Herskowitz I, Yamamoto KR: Roles of SWI1, SWI2, and SWI3 proteins for transcriptional enhancement by steroid receptors. Science 258: $1598-1604,1992$

6. Muchardt C, Yaniv M: A human homologue of Saccharomyces cerevisiae SNF2/SWI2 and Drosophila brm genes potentiates transcriptional activation by the glucocorticoid receptor. Embo J 12: 4279-4290., 1993

7. Chiba H, Muramatsu M, Nomoto A, Kato H: Two human homologues of Saccharomyces cerevisiae SWI2/SNF2 and Drosophila brahma are transcriptional coactivators cooperating with the estrogen receptor and the retinoic acid receptor. Nucleic Acids Res 22: 1815-1820., 1994

8. Ichinose H, Garnier JM, Chambon P, Losson R: Ligand-dependent interaction between the estrogen receptor and the human homologues of SWI2/SNF2. Gene 188: 95-100., 1997 
9. DiRenzo J, Shang Y, Phelan M, Sif S, Myers M, Kingston R, Brown M: BRG-1 is recruited to estrogen-responsive promoters and cooperates with factors involved in histone acetylation. Mol Cell Biol 20: 7541-7549., 2000

10. Belandia B, Orford RL, Hurst HC, Parker MG: Targeting of SWI/SNF chromatin remodelling complexes to estrogen-responsive genes. Embo J 21: 4094-4103., 2002

11. Klochendler-Yeivin A, Muchardt C, Yaniv M: SWI/SNF chromatin remodeling and cancer. Curr Opin Genet Dev 12: 73-79, 2002

12. Roberts CW, Orkin SH: The SWI/SNF complex--chromatin and cancer. Nat Rev Cancer 4: 133-142, 2004

13. Bochar DA, Wang L, Beniya H, Kinev A, Xue Y, Lane WS, Wang W, Kashanchi F, Shiekhattar R: BRCA1 is associated with a human SWI/SNF-related complex: linking chromatin remodeling to breast cancer. Cell 102: 257-265, 2000

14. Rosen EM, Fan S, Pestell RG, Goldberg ID: BRCA1 in hormone-responsive cancers. Trends Endocrinol Metab 14: 378-385, 2003

15. Narod SA, Foulkes WD: BRCA1 and BRCA2: 1994 and beyond. Nat Rev Cancer 4: 665-676, 2004

16. Futreal PA, Liu Q, Shattuck-Eidens D, Cochran C, Harshman K, Tavtigian S, Bennett LM, Haugen-Strano A, Swensen J, Miki Y, et al.: BRCA1 mutations in primary breast and ovarian carcinomas. Science 266: 120-122., 1994

17. Decristofaro MF, Betz BL, Rorie CJ, Reisman DN, Wang W, Weissman BE: Characterization of SWI/SNF protein expression in human breast cancer cell lines and other malignancies. J Cell Physiol 186: 136-145., 2001

18. Belandia B, Parker MG: Functional interaction between the p160 coactivator proteins and the transcriptional enhancer factor family of transcription factors. $\mathbf{J}$ Biol Chem 275: 30801-30805, 2000 
19. Gao X, Zacharek A, Salkowski A, Grignon DJ, Sakr W, Porter AT, Honn KV: Loss of heterozygosity of the BRCA1 and other loci on chromosome $17 \mathrm{q}$ in human prostate cancer. Cancer Res 55: 1002-1005, 1995

20. Link KA, Burd CJ, Williams E, Marshall T, Rosson G, Henry E, Weissman B, Knudsen KE: BAF57 governs androgen receptor action and androgen-dependent proliferation through SWI/SNF. Mol Cell Biol 25: 2200-2215, 2005

21. Burdall SE, Hanby AM, Lansdown MR, Speirs V: Breast cancer cell lines: friend or foe? Breast Cancer Res 5: 89-95, 2003

22. Knudson AG, Jr.: Mutation and cancer: statistical study of retinoblastoma. Proc Natl Acad Sci U S A 68: 820-823, 1971

23. Lukas J, Muller H, Bartkova J, Spitkovsky D, Kjerulff AA, Jansen-Durr P, Strauss M, Bartek J: DNA tumor virus oncoproteins and retinoblastoma gene mutations share the ability to relieve the cell's requirement for cyclin D1 function in G1. J Cell Biol 125: 625-638, 1994

24. Somboonporn W, Davis SR: Testosterone effects on the breast: implications for testosterone therapy for women. Endocr Rev 25: 374-388, 2004

25. Strobeck MW, Reisman DN, Gunawardena RW, Betz BL, Angus SP, Knudsen KE, Kowalik TF, Weissman BE, Knudsen ES: Compensation of BRG-1 function by Brm: insight into the role of the core SWI-SNF subunits in retinoblastoma tumor suppressor signaling. J Biol Chem 277: 4782-4789, 2002

26. Wang L, Baiocchi RA, Pal S, Mosialos G, Caligiuri M, Sif S: The BRG1- and hBRM-associated factor BAF57 induces apoptosis by stimulating expression of the cylindromatosis tumor suppressor gene. Mol Cell Biol 25: 7953-7965, 2005 


\section{Figure legends}

Figure 1. Identification of a CA dinucleotide insertion in exon 5 of the $B A F 57$ gene in the human BT549 breast cancer cell line. (a) The mutation was detected in two independent sequencing reactions using both the forward $(F)$ and the reverse $(\mathrm{R})$ primers. The wild-type (WT) sequence of exon 5 is given in the bottom line. The data from the sequencing reactions were processed by using the Seqman software (Macintosh). (b) Schematic representation of the restriction digestion strategy used. The $R s a$ I restriction site (GTAC) is located in the middle part of BAF57 exon 5. Digestion of the PCR-amplified wild-type exon 5 amplicon generates two DNA fragments of similar size ( $250 \mathrm{bp})$, while in the BT549-derived amplicon the CA insertion disrupts the $R s a$ I restriction site and the

original fragment remains undigested $(\sim 500 \mathrm{bp})$. (c) Restriction digestion analysis of BAF57 exon 5 with Rsa I. The restriction digestion reactions were terminated by addition of DNA loading buffer and the sizes of the digestion products were analyzed by agarose gel (1.2\%) electrophoresis. Two independently generated PCR amplicons were analyzed in each case.

Figure 2. The CA insertion present in BT549 cells generates a truncated BAF57 protein. (a) Wild-type BAF57 open reading frame. The wild-type human BAF57 protein consists of 411 amino acids. (b) The CA insertion identified in BT549 cells (indicated in capital letters) causes a frameshift leading to a premature stop codon at position 85 (indicated by an asterisk) which generates a truncated BAF57 protein consisting of the first 75 residues of BAF57 protein followed by 9 random amino acids (highlighted inside a rectangle). 
Figure 3. Comparison of the relative BAF57 expression levels in a panel of breast and prostate cancer cell lines by Real-Time RT-PCR. Total RNA was extracted from a number of breast and prostate cancer cell lines (BT549, ZR-75-1, MCF-7, T47D, LNCaP and PC-3). cDNA was generated and BAF57 mRNA expression analysed by Real-Time RT-PCR using L19 as control gene. The values are expressed relative to the BAF57 mRNA levels in BT549 cells assigned as 1.

Figure 4. Functional characterization of the BT-BAF57 truncated mutant. (a) In vitro interaction of BAF57 and BT-BAF57 with ER $\alpha$. Binding of GST fusion proteins of wild type BAF57 (GST-BAF57) or truncated BT-BAF57 (GST-BT-BAF57) to ${ }^{35}$ S-labelled $\mathrm{ER} \alpha$ in the presence of $100 \mathrm{nM} 17 \beta$-estradiol (E2) or vehicle (-). (b) In vitro interaction of BAF57 and BT-BAF57 with SRC1e. Binding of GST fusion proteins of wild type BAF57 (GST-BAF57) or BT-BAF57 (GST-BT-BAF57) to ${ }^{35}$ S-labelled SRC1 bHLH-PAS domain (amino acids 1-361). In each panel, the input lane represents $10 \%$ of the total volume of lysate used in each reaction. (c) Effect of BT-BAF57 expression on transcriptional activation by ER $\alpha$ in cells expressing endogenous BAF57. COS-1 cells were transiently transfected with expression vectors for ER $\alpha$ and SRC1e, the 2XERE-pS2 luciferase vector, the dominant negative $\triangle \mathrm{N}$-BAF57 or BT-BAF57. In each case, after transfection, cells were washed and incubated with vehicle (white bars) or $17 \beta$-estradiol (back bars) at $10^{-8}$ for 24 h. Subsequently, cell lysates were assayed using a dual luciferase reporter system. Normalized values are expressed relative to the activity of $\operatorname{ER} \alpha$ alone in the presence of ligand. The results shown represent the average of two independent experiments assayed in duplicate.

Table 1. Summary of human cancer cell lines screened

\begin{tabular}{lllll}
\hline Breast cancer cell lines & Genomic DNA & cDNA & ER status & BAF57 mutations \\
\hline
\end{tabular}




\begin{tabular}{lcccc}
\hline MCF-7 & + & - & + & - \\
ZR-75-1 & + & - & + & - \\
BT549 & + & + & - & + \\
T47D & + & - & + & - \\
MDA-MB-361 & + & - & + & - \\
MDA-MB-231 & - & + & - & - \\
\hline Prostate cancer cell lines & & & & - \\
\hline PC-3 & - & + & $+/-$ & +- \\
LNCaP & - & + & $+/-$ & \\
\hline
\end{tabular}


Figure 1

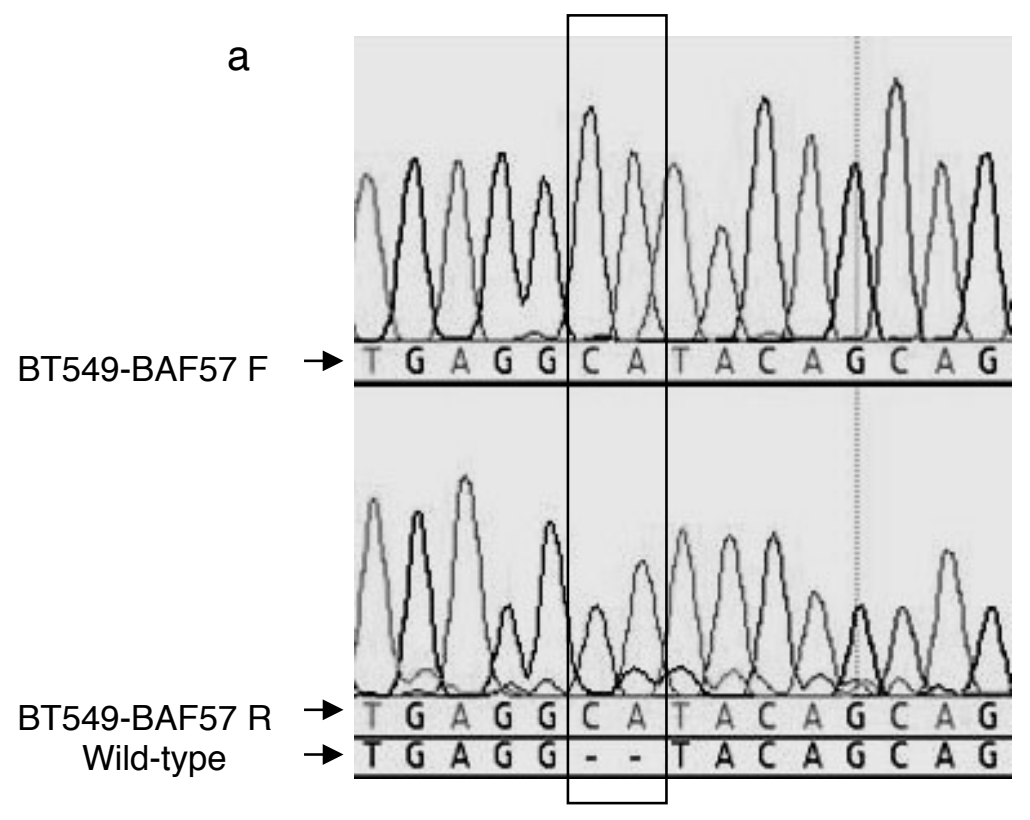

b

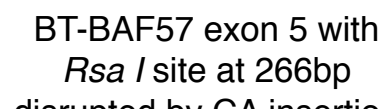
disrupted by CA insertion

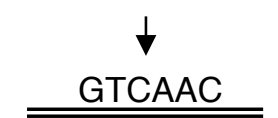

Restriction digestion with

Rsa I

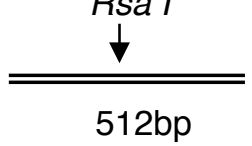

$512 b p$
WT-BAF57 exon 5 with Rsa / site at 266bp

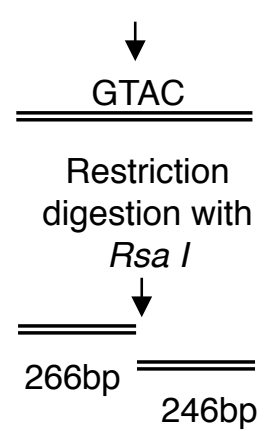

C

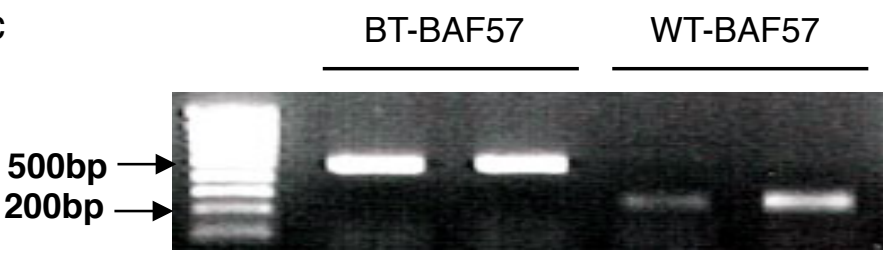


a $1 / 1 \quad 31 / 11 \quad 61 / 21$

atg tca aaa aga cca tet tat gcc cca cct ccc acc cca get cct gca aca caa atg cce agc aca cca ggg ttt gtg gga tac aat cca $\begin{array}{lllllllllllllllllllllllllllllll}\mathbf{M} & \mathbf{S} & \mathrm{K} & \mathbf{R} & \mathbf{P} & \mathbf{S} & \mathbf{Y} & \mathbf{A} & \mathbf{P} & \mathbf{P} & \mathbf{P} & \mathbf{T} & \mathbf{P} & \mathbf{A} & \mathbf{P} & \mathbf{A} & \mathbf{T} & \mathbf{Q} & \mathbf{M} & \mathbf{P} & \mathbf{S} & \mathbf{T} & \mathbf{P} & \mathbf{G} & \mathbf{F} & \mathbf{V} & \mathbf{G} & \mathbf{Y} & \mathbf{N} & \mathbf{P} & \end{array}$
$91 / 31$
$121 / 41$
$151 / 51$

tac agt cat ctc gcc tac aac aac tac agg ctg gga ggg aac ccg gge acc aac age ćgg gtc acg gca tcc tct ggt atc acg att cca $\begin{array}{llllllllllllllllllllll}\mathbf{Y} & \mathbf{S} & \mathbf{H} & \mathrm{L} & \mathbf{A} & \mathbf{Y} & \mathbf{N} & \mathbf{N} & \mathbf{Y} & \mathbf{R} & \mathbf{L} & \mathbf{G} & \mathbf{G} & \mathbf{N} & \mathbf{P} & \mathbf{G} & \mathbf{T} & \mathbf{N} & \mathbf{S} & \mathbf{R} & \mathbf{V} & \mathbf{T} \\ 181 / 61 & & & & & & & & & 211 / 71 & & & & & & & & & & \\ 241 / 81\end{array}$

aaa ccc cca aag cca cca gat aag ccg ctg atg ccc tac atg agg tac age aga aag gtc tgg gac caa gta aag gct tcc aac cct gac

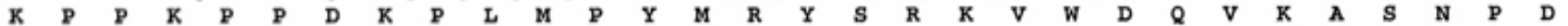

$271 / 91 \quad 301 / 101 \quad 331 / 111$

cta aag ttg tgg gag att gge aag att att ggt ggc atg tgg cga gat ctc act gat gaa gaa aaa caa gaa tat tta aac gaa tac gaa

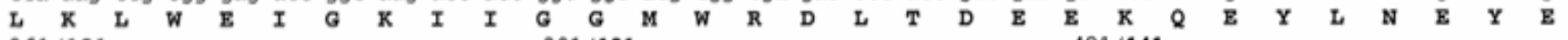
$361 / 121 \quad 391 / 131 \quad 421 / 141$

gca gaa aag ata gag tac aat gaa tct atg aag gce tat cat aat tcc cce geg tac ctt gct tac ata aat gca aaa agt cgt gca gaa $\begin{array}{llllllllllllllllllllllllllllll}\mathbf{A} & \mathbf{E} & \mathbf{K} & \mathbf{I} & \mathbf{E} & \mathbf{Y} & \mathbf{N} & \mathbf{E} & \mathbf{S} & \mathbf{M} & \mathbf{K} & \mathbf{A} & \mathbf{Y} & \mathbf{H} & \mathbf{N} & \mathbf{S} & \mathbf{P} & \mathbf{A} & \mathbf{Y} & \mathbf{L} & \mathbf{A} & \mathbf{Y} & \mathbf{I} & \mathbf{N} & \mathbf{A} & \mathbf{K} & \mathbf{S} & \mathbf{R} & \mathbf{A} & \mathbf{E}\end{array}$ $451 / 151$ $481 / 161$ $511 / 171$

get get tta gag gaa gaa agt cga cag aga caa tct cgc atg gag aaa gga gaa ccg tac atg agc att cag cet get gaa gat cca gat

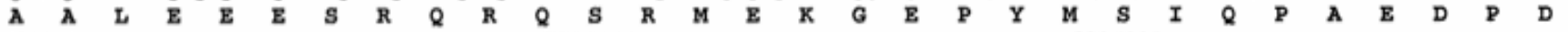
$\begin{array}{lccc}541 / 181 & 571 / 191 & 601 / 201\end{array}$

gat tat gat gat gge ttt tca atg aag cat aca gec acc gcc cgt ttc cag aga aac cac cge ctc atc agt gaa att ctt agt gag agt

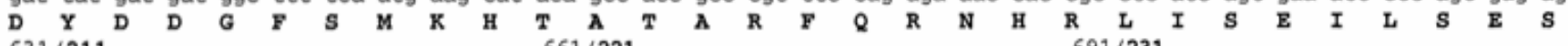
631/211 661/221 $691 / 231$

gtg gtg cca gac gtt cgg tca gtt gtc aca aca get aga atg cag gtc ctc aaa cgg cag gtc cag tcc tta atg gtt cat cag cga aaa

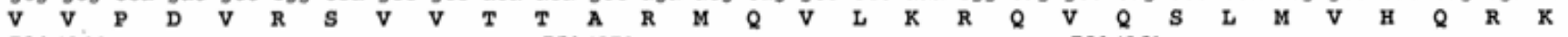

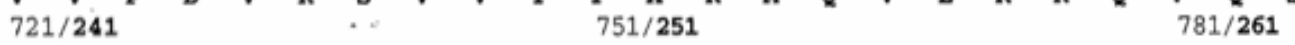
cta gaa get gaa ctt ctt caa ata gag gaa cga cac cag gag aag aag agg aaa ttc ctg gaa agc aca gat tca ttt aac aat gaa ctt

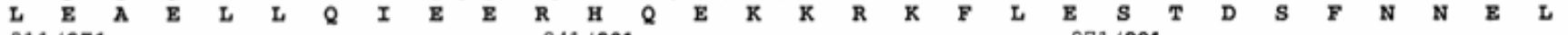
$811 / 271 \quad 841 / 281 \quad 871 / 291$

aaa agg ttg tge ggt ctg aaa gta gaa gtg gat atg gag aaa att gca get gag att gca cag gca gag gaa cag gcc cgc aaa agg cag

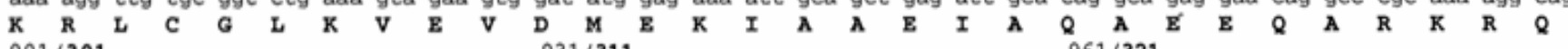
$901 / 301 \quad 931 / 311$ $961 / 321$

gag gaa agg gag aag gag gcc gca gag caa gct gag cgc agt cag agc agc atc gtt cct gag gaa gaa caa gca gct aac aaa ggc gac

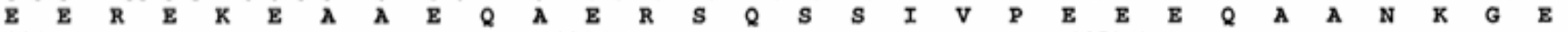
$991 / 331 \quad 1021 / 341 \quad 1051 / 351$

gag aag aaa gac gac gag aac att ceg atg gag aca gag gag aca cac ctt gaa gaa aca aca gag agc caa cag aat ggt gaa gaa ggc

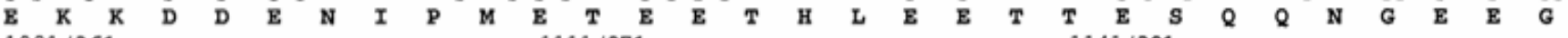
$1081 / 361 \quad 1111 / 371 \quad 1141 / 381$

acg tct act cet gag gac aag gag agt ggg cag gag ggg gtc gac agt atg gca gag gaa gga acc agt gat agt aac act ggc tcg gag

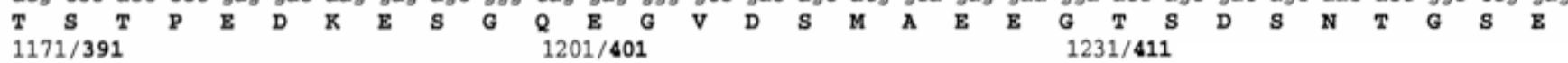
agc aac agt gea aca gtg gag gag cca cca aca gat ccc ata cca gaa gat gag aaa aaa gaa taa

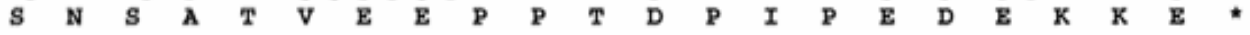

b $1 / 1 \quad 31 / 11 \quad 61 / 21$

atg tca aaa aga cca tet tat gcc cca cct ccc acc cca get cet gca aca caa atg cce age aca cca ggg ttt gtg gga tac aat cca

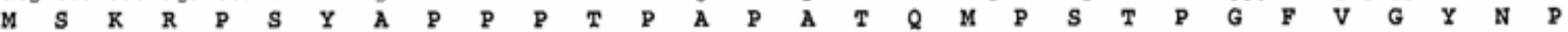
91/31 121/41 151/51

tac agt cat ctc gec tac aac aac tac agg ctg gga ggg aac ccg gge acc aac age cgg gte acg gea tce tet ggt atc acg att cca

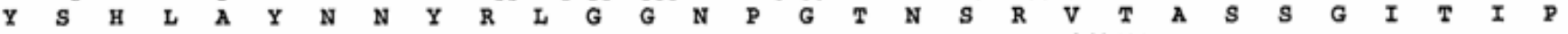
181/61 211/71 261/81

aaa cCC cca aag cca cca gat aag ccg ctg atg ccc tac atg agg CAt aca gca gaa agg tct ggg acc aag taa

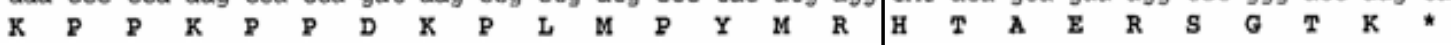


Figure 3

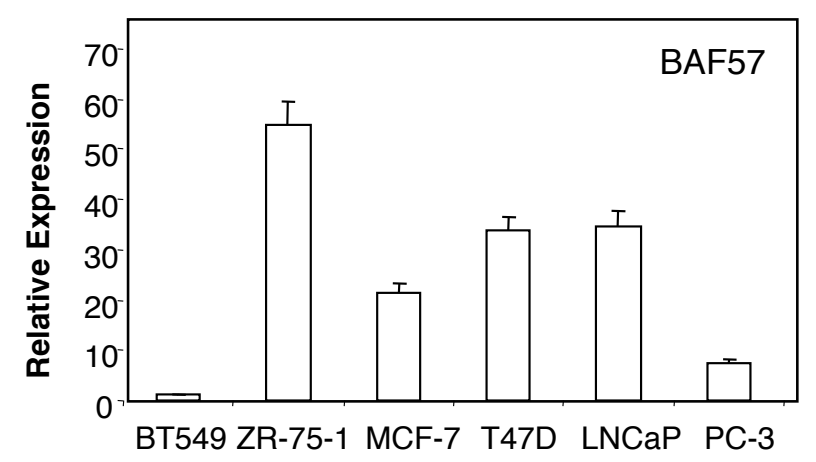


Figure 4

a

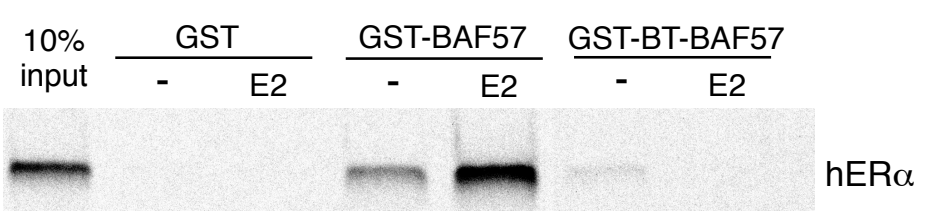

b

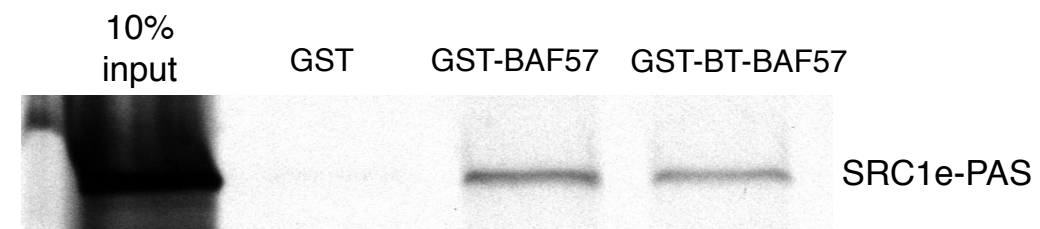

C

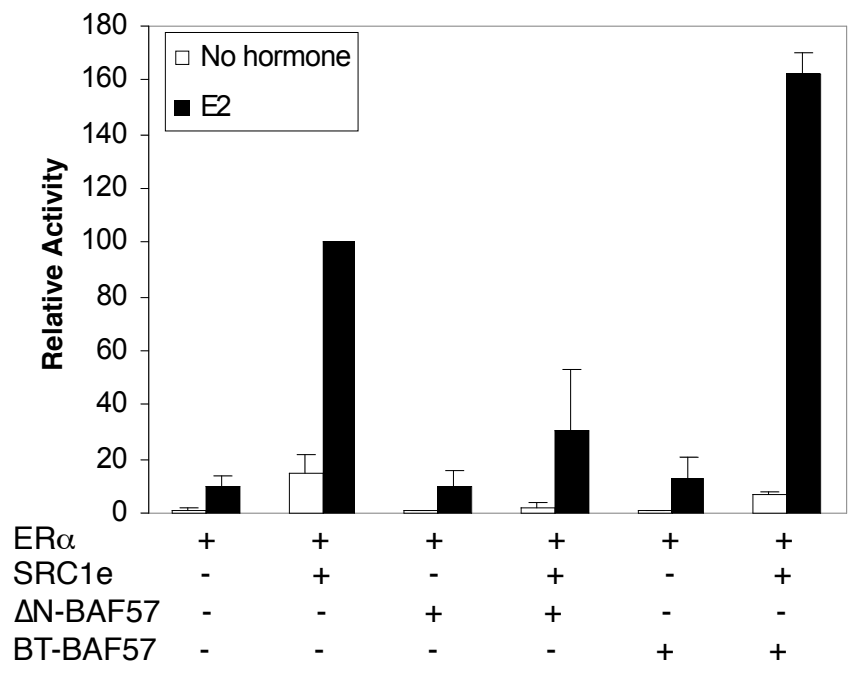

